\title{
Options for Denormal Representation in Logarithmic Arithmetic
}

\author{
Mark G. Arnold · Caroline Collange
}

the date of receipt and acceptance should be inserted later

\begin{abstract}
Economical hardware often uses a FiXed-point Number System (FXNS), whose constant absolute precision is acceptable for many signal-processing algorithms. The almostconstant relative precision of the more expensive Floating-Point (FP) number system simplifies design, for example, by eliminating worries about FXNS overflow because the range of FP is much larger than FXNS for the same wordsize; however, primitive FP introduces another problem: underflow. The Signed Logarithmic Number System (SLNS) offers similar range and precision as FP with much better performance (in terms of power, speed and area) for multiplication, division, powers and roots. This paper proposes three variations of a new number system, respectively called the Denormal LNS (DLNS), Denormal Mitchell LNS (DMLNS) and Denormal Offset Mitchell LNS (DOMLNS), which are all hybrids of the properties of FXNS and SLNS. The inspiration for $\mathrm{D}(\mathrm{OM}) \mathrm{LNS}$ comes from the denormal (aka subnormal) numbers found in IEEE-754 (that provide better, gradual underflow) and the $\mu$-law often used for speech encoding; the novel DLNS circuit here allows arithmetic to be performed directly on such encoded data. The proposed approach allows customizing the range in which gradual underflow occurs. Our first DLNS implementation leverages existing SLNS basic blocks. Synthesis shows the novel circuit primarily consists of traditional SLNS addition and subtraction tables, with additional datapaths that allow the novel arithmetic unit to act on conventional SLNS as well as DLNS and mixed data, for a worst-case area overhead of $26 \%$. Unlike SLNS, this DLNS implementation is still costly for general (non-constant) multiplication, division and roots. To overcome this difficulty, this paper proposes the other variations called Denormal Mitchell LNS (DMLNS) and Denormal Offset Mitchell LNS (DOMLNS), in which the well-known Mitchell's method makes the cost of general multiplication, division and roots closer to that of SLNS. Taylor-series computations suggest subnormal values in DMLNS and DOMLNS also behave similarly to those in the IEEE-754 FP standard. Synthesis shows that DMLNS and DOMLNS respectively have average area overheads of $25 \%$ and $17 \%$ compared to an equivalent SLNS 5-operation unit.
\end{abstract}

M. G. Arnold

XLNS Research, PO Box 605 Laramie WY 82070 USA, E-mail: markgarnold@xlnsresearch.com

C. Collange

INRIA, Centre de recherche Rennes - Bretagne Atlantique, Rennes, France, E-mail: caroline.collange@inria.fr 
Keywords: Computer Arithmetic, Logarithmic Number Systems (LNS), underflow, denormal, subnormal.

\section{Introduction}

Designers of application-specific systems often have knowledge about their numeric requirements, which can be satisfied with more economical arithmetic circuits than found in general-purpose systems. This has given rise to a variety of special-purpose number systems, that have certain advantages in application-specific systems. For example, designers may know that most numbers processed by the application fall within a certain range; neglecting an occasional small number that underflows this range gives only a small error acceptable to the application. This paper considers a new number system, which combines features of several well-known number systems, to give application-specific designers new options for dealing with such situations, particularly in applications like signal-processing.

In computer arithmetic, a representation (denoted in uppercase: $X$ ) is a finite vector of bits that represents a numeric value in a particular number system. The value (lowercase $x$ ) is a real number that may be approximated by $X$. There is a particular real, $\bar{x}$, that $X$ represents exactly. Other values of $x$ in the neighborhood of $\bar{x}$ use the same representation. The resulting error can be measured in bits of absolute error (1), or in bits of relative error (2):

$$
\begin{gathered}
e_{a}=\log _{2}|x-\bar{x}| \\
e_{r}=\log _{2}\left(\left|\frac{x-\bar{x}}{\bar{x}}\right|\right)=\log _{2}|1-x / \bar{x}| .
\end{gathered}
$$

The simplest number system of this kind is the fixed-point number system, in which $X$ consists of a signed integer $X_{F}$ that is scaled by $2^{F}$ to provide a constant $F$ bits of absolute precision:

$$
\bar{x}=X_{F} \cdot 2^{-F} .
$$

Many problems perform better when the relative precision is held constant. Binary floating-point number systems provide nearly constant relative precision by providing

$$
\bar{x}=(-1)^{X_{S}} X_{M} 2^{X_{E}}
$$

where $X$ is subdivided into three parts: the sign $\left(X_{S} \in\{0,1\}\right)$, the fixed-point mantissa $\left(1 \leq X_{M}<2\right)$ and the integer exponent $\left.X_{E}=\left\lfloor\log _{2}|x|\right\rfloor\right)$. The choice of these fields impacts the quality of the floating-point system. Using hidden-bit normalization, there can be an assumed 1 in $X_{M}=1+X_{F} \cdot 2^{-F}$. Because of the finite size of $X$, there are upper and lower bounds on the exponent, $L \leq X_{E}<U$, which determines the dynamic range, $2^{L} \leq \bar{x}<2^{U}$.

To overcome incompatibility caused by different manufacturer's arbitrary choices for $X_{M}$ and $X_{E}$, a formal standard for binary floating-point, IEEE-754 [15], was adopted quickly in the 1980s by all manufacturers, and was revised in 2008 [16]. IEEE-754 uses single (32-bit $X, F=23, L=-126$ and $U=128$ ) and double (64-bit $X, F=52$, $L=-1022$ and $U=1024$ ) precision, named Binary32 and Binary64, respectively, in the 2008 standard, with hidden-bit normalization. IEEE 754 actually encodes $X_{E}$ with a biased exponent, but that is irrelevant for the discussion of what values can be represented.

One of the features introduced in IEEE 754, which was controversial at the time, is gradual underflow, sometimes called subnormals or denormals. Prior to IEEE 754, most floating point systems left a gap between the smallest representable positive number, $2^{L}$, and 
zero. There would be a similar gap on the negative side. To fill this gap, IEEE 754 defines a special case (signaled here by $X_{E}=L-1$ ) where an unnormalized $X_{M}=X_{F} \cdot 2^{-F}$ has the same meaning as a fixed point value between zero and $2^{L}$, in other words:

$$
\bar{x}=(-1)^{X_{S}} X_{M} 2^{L-1}
$$

when $X_{E}=L-1$. The value +0.0 is then not a unique case, but rather just the nonnegative subnormal with the smallest absolute value, defined by $X_{M}=0$ and $X_{S}=0$. IEEE 754 requires a distinct representation of -0.0 , similarly defined as the subnormal with $X_{M}=0$ and $X_{S}=1$.

In 1975, Swartzlander and Alexopoulos [27] proposed the Signed Logarithm Number System (SLNS), which represents the magnitude of values with their base- $b$ logarithms and a concatenated sign bit. SLNS represents a real number, $x$, using a sign bit, $X_{S}$, and a finite approximation to the logarithm of the absolute value, $X_{L}=Q\left(2^{F} \cdot \log _{b}|x|\right) / 2^{F}$, where $F$ is the precision and $Q$ is a quantization function whose output (defining a particular rounding mode) is an integer that fits within the finite word. A given SLNS representation, defined by $X_{S}$ and $X_{L}$, maps into the exact value

$$
\bar{x}=(-1)^{X_{S}} \cdot b^{X_{L}} .
$$

With the typical choice of $b=2$ and a symmetrical range of exponents $(L \approx-U)$, the dynamic range (including non-denormal underflow) is similar to floating point, since $L \leq X_{L}<U$. SLNS keeps this logarithmic representation during all computation (including addition). When precision requirements are low to moderate and multiplication is more frequent than addition, SLNS is more cost effective than floating point. The simplest definition of SLNS excludes representing an exact zero; a special bit may be included to allow for this at some extra hardware cost.

An isomorphic definition of SLNS [23] uses integer powers of the smallest value greater than 1.0 that is exactly representable, $\beta=b^{2^{-F}}$. With either definition, the relative spacing between SLNS points is $\beta$, and with faithful rounding [3] when $|x|$ is larger than $|\bar{x}|,|x / \bar{x}| \leq$ $\beta$. The relative precision is $1-\beta$ and from (2), the number of bits of relative precision will be the constant $\log _{2}(1-\beta) \approx F$.

Multiplication and division are straightforward in SLNS. Since the values are already represented as logarithms, a simple addition or subtraction computes the product or quotient, together with an exclusive OR to find the sign. Although it makes multiplication and division easy, SLNS makes addition and subtraction more difficult than fixed point. The manual algorithm for logarithmic addition was first described by Leonelli and popularized by Gauss in the early nineteenth century [14]. Swartzlander et al. [27,26] and others [19, 12] reconsidered these algorithms and found them quite attractive in light of the technology available for digital signal processing in the 1970s. Beyond simple table lookup, several implementations $[21,8,7,6]$ have provided SLNS arithmetic with increased performance and reduced implementation cost. In particular SLNS appears to offer reduced power consumption in many applications [25,23]. Successful applications have included massive scientific simulation [24], Hidden-Markov Models (HMM) [28], and music synthesis [20]. The European Logarithmic Microprocessor (ELM) [9] provides dual SLNS Arithmetic and Logic Units (ALUs) that implement the Gauss/Leonelli algorithm in $0.18 \mu \mathrm{m} 125 \mathrm{MHz}$ hardware. More recently, advances in FPGA [13] and cotransformation [17] implementations of SLNS allow higher-precision applications to be affordable. Logarithmic arithmetic has generalizations in the complex numbers [4] and quaternions [5]. 
The Gauss/Leonelli addition algorithm requires computing one of the two following functions. When the signs of the numbers to be added are the same, the hardware computes

$$
s_{b}(z)=\log _{b}\left(1+b^{z}\right) .
$$

For all possible $z, 0<s_{b}(z)$. For $z>0, s_{b}(z) \geq z$. It is not necessary for the hardware to deal with both positive and negative $z$ since

$$
s_{b}(z)=s_{b}(-z)+z .
$$

When the signs of the numbers are different, the hardware computes

$$
d_{b}(z)=\log _{b}\left|1-b^{z}\right|
$$

For $z \geq \log _{b}(2), 0 \leq d_{b}(z)<z$. Analogously to $s_{b}$,

$$
d_{b}(z)=d_{b}(-z)+z .
$$

There is a point, $E_{0} \approx F$, known as the essential zero, for $z<-E_{0}$ where $s_{b}(z)<2^{-F}$ and $d_{b}(z)<2^{-F}$, in other words, the quantized values are zero. From (7) and (9), there is a similar essential-identity property $\left(s_{b}(z) \approx d_{b}(z) \approx z\right)$ for $z>E_{0}$.

Given $\bar{x}$ represented as $X_{S}$ and $X_{L}$, and $\bar{y}$ represented as $Y_{S}$ and $Y_{L}$, there are two cases for SLNS addition. If $X_{S}=Y_{S}$,

$$
\begin{aligned}
\bar{x}+\bar{y} & =(-1)^{R_{S}} \cdot\left(b^{X_{L}}+b^{Y_{L}}\right) \\
& =(-1)^{R_{S}} \cdot\left(b^{X_{L}} \cdot\left(1+b^{Y_{L}} / b^{X_{L}}\right)\right) \\
& =(-1)^{R_{S}} \cdot b^{R_{L}},
\end{aligned}
$$

where the actual computation performed by the hardware is

$$
R_{L}=X_{L}+s_{b}\left(Y_{L}-X_{L}\right) .
$$

If $X_{S} \neq Y_{S}$, the hardware does a similar computation,

$$
T_{L}=X_{L}+d_{b}\left(Y_{L}-X_{L}\right) .
$$

(The variables $P \ldots T$ will be reserved for results in this paper.) The sign of the result $\left(R_{S}\right.$ or $T_{S}$ ) is simply the sign of the larger of the input arguments.

An earlier attempt to incorporate denormals into SLNS [2] is quite different than what is proposed in this paper. Arnold et al. [2] treat denormals specially and use over a dozen cases to consider operands and results of different magnitudes. In contrast, the novel representation proposed here may accomplish similar gradual underflow using simple algorithms that do not explicitly refer to the magnitude of the operands or results. The simple algorithms proposed here will be much more efficient than those of [2] in a software-based gradual-underflow implementation (for instance, on the the ELM $[9,17]$, a microprocessor that provides hardware for SLNS-without-denormals). Furthermore, while [2] only applies to denormals patterned after IEEE-754, the novel approach in this paper suggests a range of denormal representations (from one similar to IEEE-754 to a fully-denormal one similar to the $\mu$-law for speech encoding [30]).

This paper is an extended version of [47], which introduced the original DLNS system. In addition to DLNS, we present here two novel number systems, DMLNS and DOMLNS, which are variations of the DLNS system that enable different trade-offs between cost and 
accuracy. The present paper considers two additional Taylor series to evaluate the accuracy of the three systems, DLNS, DMLNS and DOMLNS, and gives a more precise description of the computations performed. Finally, it evaluates the hardware cost in area and latency of DMLNS and DOMLNS by presenting synthesis results of a 5-operation ALU on FPGAs.

Section 2 describes the novel DLNS representation and gives options for how DLNS-toDLNS addition may be performed (and noting that other general DLNS-by-DLNS, like multiplication, are expensive). Section 3 considers simplifications possible when not all operands are given in DLNS. Section 4 presents a simple model for DLNS error, and observes this model roughly predicts the errors we observe with actual DLNS arithmetic in simulation of a typical application, the Fast Fourier Transform (FFT). This section also reports DLNS may reduce bit-level switching activity (and therefore power consumption) for the FFT. Section 5 presents synthesis results for the preferred DLNS circuit. Section 6 presents two alternative implementation called Denormal Mitchell LNS (DMLNS) and Denormal Offset Mitchell LNS (DOMLNS) that lower the cost of general multiplication. Section 7 presents conclusions.

\section{DLNS-to-DLNS Operations}

The Denormal Logarithmic Number System (DLNS) uses a compression function, $f$, and a decompression function, $f^{-1}$, to convert into and out of SLNS. The value, $x$, represented by a denormal representation, $\left(X_{S}, X_{D}\right)$, is the value represented by its decompression into SLNS, $\left(X_{S}, f^{-1}\left(X_{D}\right)\right)$ :

$$
\bar{x}=(-1)^{X_{S}} \cdot\left(b^{f^{-1}\left(X_{D}\right)}\right) .
$$

There is some lattitude in the definition of this pair of functions, but whatever their definition, they must be (as close as possible to) exact inverses of each other, $f\left(f^{-1}(x)\right)=x$. This paper will consider three different ways to define this pair of functions. In this section, we will consider how to define this pair of functions so that the same kind of hardware function unit that computes SLNS can also be used for all DLNS operations. The approach in this section simplifies nicely for some cases (described in Section 3), but is expensive for others (such as DLNS-by-DLNS multiplication and division). In section 6, we consider alternative definitions for this pair of functions, which do not allow for that kind of simplification, but whose hardware cost is much lower (closer to the classical SLNS advantage of cheap multiplication and division).

For the moment we will define

$$
\begin{array}{r}
f(x)=s_{b}(x-J)+J, \\
f^{-1}(x)=d_{b}(x-J)+J,
\end{array}
$$

which allows (12) to be restated as:

$$
\bar{x}=(-1)^{X_{S}} \cdot\left(b^{X_{D}}-b^{J}\right),
$$

where $J \leq X_{D}<U$ and $J \leq 0$ is an integer constant for implementation convenience. Notice that, unlike simple SLNS, DLNS does not need a special bit to represent zero exactly, but rather uses $X_{D}=J$. DLNS has some similarity to redundant LNS [1] and multidimensional LNS [22] that involve a definition with addition/subtraction of two exponentials; however unlike those systems, in DLNS one of the exponentials is a constant. The 
choice of the constant $J$ in (15) is arbitrary; a large negative $J$ restricts the denormal behavior to values close to zero (analogous to IEEE-754); $J$ near 0 makes DLNS like FXNS.

Compared to the symmetrical SLNS representation, where $L \leq X_{L}<U$, DLNS (with the choice of $J=0$ in (15)) typically requires one fewer bit than SLNS. DLNS does this at the cost of reducing the relative precision for values near zero. In effect, values near zero are represented with $F$-bit absolute precision (similar to FXNS); values far from zero are represented with $F$-bit relative precision (similar to FP and conventional SLNS).

This section describes cases when all the inputs and outputs are in pure-DLNS format. The next section will consider how the cases simplify when some of the inputs are not in pure-DLNS format. In this section, we consider a dyadic DLNS-to-DLNS operation op ${ }_{D}$ with two operands, $\left(X_{S}, X_{D}\right)$ and $\left(Y_{S}, Y_{D}\right)$, that produces a result $\left(R_{S}, R_{D}\right)$. Conceptually, the approach is to convert $X_{D}$ and $Y_{D}$ into $X_{L}=f^{-1}\left(X_{D}\right)$ and $Y_{L}=f^{-1}\left(Y_{D}\right)$ and then perform

$$
R_{D}=f\left(\mathrm{op}_{L}\left(X_{L}, Y_{L}\right)\right),
$$

where $\mathrm{op}_{L}$ is the equivalent SLNS operation, for example op ${ }_{L}\left(X_{L}, Y_{L}\right)=X_{L}+Y_{L}$ for multiplication. With (13) and (14) the cost of DLNS-by-DLNS multiplication or division is rather expensive: two $d_{b}$ and one $s_{b}$ units; however, the next subsections illustrate how the cost of DLNS-to-DLNS addition may be simplified.

\subsection{DLNS-to-DLNS Addition}

The problem of DLNS addition is to find the closest representation to

$$
\bar{x}+\bar{y}=(-1)^{X_{S}} \cdot\left(b^{X_{D}}-b^{J}\right)+(-1)^{Y_{S}} \cdot\left(b^{Y_{D}}-b^{J}\right) .
$$

Just as with conventional SLNS, the hardware has to deal with two cases, a) when the signs of $\bar{x}$ and $\bar{y}$ are the same, and b) when the signs are different (in other words, $X_{S}=Y_{S}$ and $\left.X_{S} \neq Y_{S}\right)$.

\subsection{Same Signs}

Suppose $\bar{x}$ and $\bar{y}$ have the same sign. The sign of the result, $R_{S}=X_{S}=Y_{S}$, will be the same, which allows the sign to be factored out of the computation of the magnitude of the result. There are two alternative ways to derive the computation that the DLNS hardware performs. The first of these performs the addition first, and then converts this back to the DLNS format:

$$
\begin{aligned}
\bar{x}+\bar{y} & =(-1)^{R_{S}} \cdot\left(\left(\left(b^{X_{D}}+b^{Y_{D}}\right)-b^{J}\right)-b^{J}\right) \\
& =(-1)^{R_{S}} \cdot\left(\left(b^{X_{D}}\left(1+b^{Y_{D}} / b^{X_{D}}\right)-b^{J}\right)-b^{J}\right) \\
& =(-1)^{R_{S}} \cdot\left(\left(b^{\log _{b}\left(b^{X_{D}}\left(1+b^{Y_{D}} / b^{X_{D}}\right)\right)}-b^{J}\right)-b^{J}\right) \\
& =(-1)^{R_{S}} \cdot\left(\left(b^{X_{D}+s_{b}\left(Y_{D}-X_{D}\right)}-b^{J}\right)-b^{J}\right) \\
& =(-1)^{R_{S}} \cdot\left(b^{R_{D}}-b^{J}\right)
\end{aligned}
$$


where the actual computation performed by the hardware in this case,

$$
\begin{aligned}
R_{D} & =\log _{b}\left(b^{X_{D}+s_{b}\left(Y_{D}-X_{D}\right)}-b^{J}\right) \\
& =J+d_{b}\left(X_{D}+s_{b}\left(Y_{D}-X_{D}\right)-J\right),
\end{aligned}
$$

uses both $s_{b}$ and $d_{b}$. The commutativity of addition allows interchanging $X_{D}$ and $Y_{D}$ and we can make the argument to $d_{b}$ positive. If $X_{D}>E_{0}+J \approx F+J$, we know (since $s_{b}$ is always positive) that $X_{D}+s_{b}\left(Y_{D}-X_{D}\right)-J>E_{0}$ and that the $d_{b}$ is an essential identity. In that case, this leaves $R_{D}=J+X_{D}+s_{b}\left(Y_{D}-X_{D}\right)-J=X_{D}+s_{b}\left(Y_{D}-X_{D}\right)$, in other words, the standard SLNS addition algorithm. Just like IEEE-754 (or the messy LNS algorithms in [2] inspired by it), the simple algorithm (17) maintains constant relative precision, except for gradual underflow of "tiny" numbers. The distinction here is that the definition of "tiny" is user configurable with the choice of $F$ and $J$.

The alternative approach (still for the case when the signs of $\bar{x}$ and $\bar{y}$ are the same) converts one of the representations to SLNS before performing the addition:

$$
\begin{aligned}
\bar{x}+\bar{y} & =(-1)^{R_{S}} \cdot\left(\left(b^{X_{D}}-b^{J}\right)+\left(b^{Y_{D}}-b^{J}\right)\right) \\
& =(-1)^{R_{S}} \cdot\left(\left(b^{X_{D}}+\left(b^{Y_{D}}-b^{J}\right)\right)-b^{J}\right) \\
& =(-1)^{R_{S}} \cdot\left(b^{R_{D}^{\prime}}-b^{J}\right)
\end{aligned}
$$

where the actual computation performed by the hardware in this case is

$$
\begin{aligned}
R_{D}^{\prime} & =\log _{b}\left(\left(b^{X_{D}}+\left(b^{Y_{D}}-b^{J}\right)\right)\right. \\
& =X_{D}+s_{b}\left(J+d_{b}\left(Y_{D}-J\right)-X_{D}\right) .
\end{aligned}
$$

(18) also uses both $s_{b}$ and $d_{b}$, although in the opposite order from (17). The argument to $d_{b}$ is positive, unless $Y_{D}=J$ (which represents $\bar{y}=0.0$ ). Since $d_{b}$ has a singularity, the hardware that computes (18) must return $R_{D}^{\prime}=X_{D}$ in that case. By similar reasoning as with the other alternative, if $Y_{D}>E_{0}+J \approx F+J$, (18) reduces to the standard SLNS addition algorithm. Given that in DLNS, $X_{D} \geq J$ and $Y_{D} \geq J$, the two alternatives produce the same result in all cases, $R_{D}=R_{D}^{\prime}$, assuming that $s_{b}$ and $d_{b}$ could be computed precisely.

\subsection{Different Signs}

The other case for DLNS addition we must consider is when $\bar{x}$ and $\bar{y}$ have different signs. The sign of the result, $T_{S}$, will be the sign of the larger value, which we will assume is $\bar{x}$, i.e., $T_{S}=X_{S}$ and $Y_{S}$ will be the opposite of $T_{S}$. Again, there are two ways to derive the computation carried out by the hardware. We could perform the addition of opposite signs (i.e., subtraction of absolute values) first, and then convert this back to the DLNS format:

$$
\begin{aligned}
\bar{x}+\bar{y} & =(-1)^{T_{S}} \cdot\left(\left(b^{X_{D}}-b^{J}\right)-\left(b^{Y_{D}}-b^{J}\right)\right) \\
& =(-1)^{T_{S}} \cdot\left(\left(b^{X_{D}}-b^{Y_{D}}+b^{J}\right)-b^{J}\right) \\
& =(-1)^{T_{S}} \cdot\left(\left(b^{X_{D}}\left|1-\frac{b^{Y_{D}}}{b^{X_{D}}}\right|+b^{J}\right)-b^{J}\right) \\
& =(-1)^{T_{S}} \cdot\left(\left(b^{\log _{b}\left(b^{X_{D}} \mid 1-b^{Y_{D}-X_{D}}\right)}+b^{J}\right)-b^{J}\right) \\
& =(-1)^{T_{S}} \cdot\left(\left(b^{X_{D}+d_{b}\left(Y_{D}-X_{D}\right)}+b^{J}\right)-b^{J}\right) \\
& =(-1)^{T_{S}} \cdot\left(b^{T_{D}}-b^{J}\right)
\end{aligned}
$$


where the actual computation performed by the hardware in this case is

$$
\begin{aligned}
T_{D} & =\log _{b}\left(b^{X_{D}+d_{b}\left(Y_{D}-X_{D}\right)}+b^{J}\right) \\
& =J+s_{b}\left(X_{D}+d_{b}\left(Y_{D}-X_{D}\right)-J\right) .
\end{aligned}
$$

If $X_{D}>E_{0}+J \approx F+J$, (19) reduces to the standard SLNS algorithm for absolute subtraction.

The other alternative for differing signs is:

$$
\begin{aligned}
\bar{x}+\bar{y} & =(-1)^{T_{S}} \cdot\left(\left(b^{X_{D}}-b^{J}\right)-\left(b^{Y_{D}}-b^{J}\right)\right) \\
& =(-1)^{T_{S}} \cdot\left(\left|\left(b^{X_{D}}+b^{J}\right)-b^{Y_{D}}\right|-b^{J}\right) \\
& =(-1)^{T_{S}} \cdot\left(b^{Y_{D}}\left|1-\frac{b^{\left(J+s_{b}\left(X_{D}-J\right)\right)}}{b^{Y_{D}}}\right|-b^{J}\right) \\
& =(-1)^{T_{S}} \cdot\left(b^{T_{D}^{\prime}}-b^{J}\right)
\end{aligned}
$$

where the actual computation performed by the hardware in this case,

$$
T_{D}^{\prime}=Y_{D}+d_{b}\left(J+s_{b}\left(X_{D}-J\right)-Y_{D}\right),
$$

is similar to $R_{D}^{\prime}$, except the roles of $X_{D}$ and $Y_{D}$ as well as $s_{b}$ and $d_{b}$ have interchanged. Assuming that $s_{b}$ and $d_{b}$ could be computed precisely, $T_{D}=T_{D}^{\prime}$. From the above, there are four alternative combinations $\left(R_{D} / T_{D}, R_{D} / T_{D}^{\prime}, R_{D}^{\prime} / T_{D}\right.$ or $\left.R_{D}^{\prime} / T_{D}^{\prime}\right)$ of hardware possible.

\section{Mixed DLNS Operations}

It is apparent from the previous section that DLNS addition involves conversion of one number (either one of the operands or the result) from DLNS format to the conventional SLNS representation. If one of the operands is already available in SLNS format, the operations may simplify.

\subsection{DLNS plus SLNS Add}

Suppose that rather than to start with two given DLNS inputs $\left(X_{D}\right.$ and $\left.Y_{D}\right)$, the addition hardware inputs are $X_{D}$ and $Y_{L}$, the latter being the conventional SLNS representation of $\bar{y}$. The desired result is then simpler for the $X_{S}=Y_{S}$ case,

$$
\begin{aligned}
\bar{x}+\bar{y} & =(-1)^{R_{S}} \cdot\left(\left(b^{X_{D}}-b^{J}\right)+b^{Y_{L}}\right) \\
& =(-1)^{R_{S}} \cdot\left(b^{X_{D}}+b^{Y_{L}}\right)-b^{J} \\
& =(-1)^{R_{S}} \cdot\left(b^{R_{D}^{\prime}}-b^{J}\right),
\end{aligned}
$$

as is the actual computation performed by the hardware,

$$
R_{D}^{\prime \prime}=\log _{b}\left(b^{X_{D}}+b^{Y_{L}}\right)=X_{D}+s_{b}\left(Y_{L}-X_{D}\right) .
$$

More importantly, this (DLNS+SLNS yields DLNS) case is identical to what would have happened for the conventional (SLNS+SLNS yields SLNS) case. 
In a similar way, when $X_{S} \neq Y_{S}$, the hardware computation for the DLNS+SLNS yields DLNS case is:

$$
T_{D}^{\prime \prime}=X_{D}+d_{b}\left(Y_{L}-X_{D}\right) .
$$

This also identical to what would have happened for the conventional (SLNS+SLNS yields SLNS) case when $X_{S} \neq Y_{S}$.

\subsection{DLNS by SLNS Multiply}

Multiplication of two DLNS values is a difficult operation involving conversion of both operands; it is better if one of the operands can already be in SLNS format. In many signalprocessing systems, the multiplier is either constant or is reused many times (and may be brought into a register). As with SLNS, the sign of the product is simply the exclusive OR or the input sign bits. Assuming $W_{L}$ is the SLNS multiplier, and $Y_{D}$ is the DLNS multiplicand,

$$
\begin{aligned}
|\bar{w} \cdot \bar{y}| & =b^{W_{L}}\left(b^{Y_{D}}-b^{J}\right) \\
& =b^{W_{L}} b^{J+d_{b}\left(Y_{D}-J\right)}+b^{J}-b^{J} \\
& =\left(b^{W_{L}+J+d_{b}\left(Y_{D}-J\right)}+b^{J}\right)-b^{J} \\
& =b^{P_{D}}-b^{J}
\end{aligned}
$$

where the hardware computation,

$$
P_{D}=J+s_{b}\left(W_{L}+d_{b}\left(Y_{D}-J\right)\right)
$$

seems similar to the computations required for DLNS+DLNS yields DLNS cases described in Section 2.

\subsection{A combined DLNS/SLNS ALU}

The similarity of (23) to the computations in Section 2 suggests that a single ALU design could have the ability to perform pure-DLNS addition/subtraction, DLNS-by-SLNS multiplication, as well as pure-SLNS addition/subtraction. Trying to combine all of these into a single circuit will suggest that some of the alternatives described in Section 2 are less efficient than others. For example, when merging $R$ and $T^{\prime}$ into a single circuit, it is not possible to implement (23) easily with that circuit. The $R / T$ and $R^{\prime} / T^{\prime}$ combinations have an undesirable structure $\left(s_{b}\right.$ and $d_{b}$ units whose inputs and outputs are connected to multiplexors with the complication that one input of each input multiplexor is connected to the output multiplexor). This statically appears to be a feedback path requiring a register, although dynamically it resolves to be combinatorial logic (rather like the behavior of an end-aroundcarry adder). While these $R / T$ or $R^{\prime} / T^{\prime}$ circuit combinations could work, the false path will complicate use of synthesis tools. This leaves the preferred combination of $R^{\prime}$ from (18) and $T$ from (19), which is implemented by the circuit in Figure 1. Table 1 gives the select inputs to the multiplexors that allow this one circuit to compute $R^{\prime}, T, P, R^{\prime \prime}$ and $T^{\prime \prime}$. 


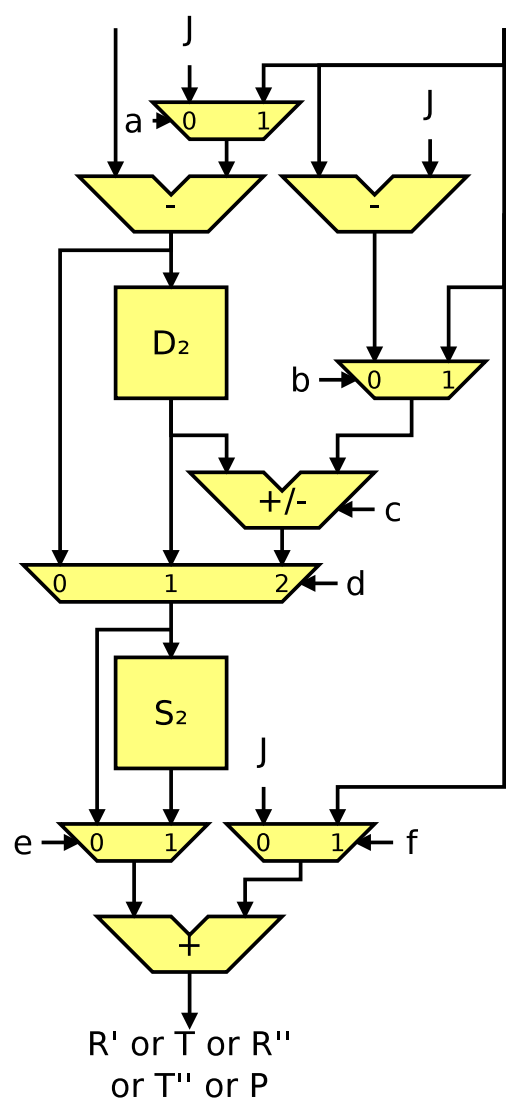

Fig. 1 DLNS ALU.

Table 1 Value of control signals as a function of the desired output. "X" stands for any value (don't care).

\begin{tabular}{||l|ccccc||}
\hline \hline Signal & $R_{D}^{\prime}$ & $T_{D}$ & $R_{D}^{\prime \prime}$ & $T_{D}^{\prime \prime}$ & $P_{D}$ \\
\hline $\mathrm{a}$ & 0 & 1 & 1 & 1 & 0 \\
$\mathrm{~b}$ & 0 & 0 & $\mathrm{X}$ & $\mathrm{X}$ & 1 \\
$\mathrm{c}$ & - & + & $\mathrm{X}$ & $\mathrm{X}$ & + \\
$\mathrm{d}$ & 2 & 2 & 0 & 1 & 2 \\
$\mathrm{e}$ & 1 & 1 & 1 & 0 & 1 \\
$\mathrm{f}$ & 1 & 0 & 1 & 1 & 0 \\
\hline
\end{tabular}

\subsection{DLNS by SLNS Multiply/Accumulate}

The three-operand multiply-accumulate operation, $w \cdot y+x$, is common in many applications. In signal processing, it frequently occurs in situations where the same $w$ is used with different values of $x$ and $y$, suggesting $w$ could be stored in SLNS format, with $x$ and $y$ in DLNS format. In this case, treating multiply-accumulate as an atomic operation (rather than 
as a multiply followed by an addition) allows considerable simplification:

$$
\begin{aligned}
|\bar{w} \cdot \bar{y}+\bar{x}| & =b^{W_{L}} \cdot\left(b^{Y_{D}}-b^{J}\right)+\left(b^{X_{D}}-b^{J}\right) \\
& =\left(b^{J+W_{L}+d_{b}\left(Y_{D}-J\right)}+b^{X_{D}}\right)-b^{J} .
\end{aligned}
$$

As with pure-DLNS addition, there are two cases, depending on signs. If the sign of $\bar{w} \cdot \bar{y}$ is the same as the sign of $\bar{x}$, the result is

$$
\begin{aligned}
|\bar{w} \cdot \bar{y}+\bar{x}| & =b^{X_{D}} \cdot\left(\frac{b^{J+W_{L}+d_{b}\left(Y_{D}-J\right)}}{b^{X_{D}}}+1\right)-b^{J} \\
& =b^{P_{D}}-b^{J}
\end{aligned}
$$

where the hardware computation is

$$
P_{D}^{\prime}=X_{D}+s_{b}\left(J+W_{L}+d_{b}\left(Y_{D}-J\right)-X_{D}\right) .
$$

If the sign of $\bar{w} \cdot \bar{y}$ is different than the sign of $\bar{x}$, the hardware computation is

$$
Q_{D}=X_{D}+d_{b}\left(J+W_{L}+d_{b}\left(Y_{D}-J\right)-X_{D}\right) .
$$

\section{Analysis and Simulation}

Unlike SLNS, the relative precision in DLNS varies with the magnitude of the value being represented in relation to the designer's choice of $b^{J}$. Given one exactly-represented-DLNS point, $|\bar{x}|$, the internal value processed by logarithmic hardware would look like $|\bar{x}|+b^{J}$. Such internal hardware is subject to the same relative spacing as conventional $F$-bit SLNS, and so the value of the next larger exactly-represented-DLNS point is $\beta\left(|\bar{x}|+b^{J}\right)-b^{J}$. From this we see the absolute spacing of the adjacent points is $(\beta-1)\left(|\bar{x}|+b^{J}\right)$ and for $|\bar{x}| \geq b^{J}$ the relative spacing is

$$
\frac{(\beta-1)\left(|\bar{x}|+b^{J}\right)}{|\bar{x}|}
$$

For $|\bar{x}|<b^{J}$, DLNS naturally underflows to the representation $|\bar{x}|=0.0$, and hence (26) is undefined.

The Fast Fourier Transform (FFT) is a common signal-processing algorithm, often implemented with both fixed- and floating-point arithmetic. It has also been extensively studied in the context of SLNS $[26,18,3,13]$. We implemented an FFT using actual SLNS (with a wide enough dynamic range that underflow does not occur) and our proposed DLNS $b=2$ arithmetics. Figure 2 shows the RMS error for a 64-point radix-two FFT whose input is a real-valued $25 \%$ duty-cycle square wave plus complex white noise. (We obtained similar figures for larger size FFTs.) This code was simulated 100 times with different pseudorandom noise. Using the same initial random data, the simulation computes several results: a double precision result which, for practical purposes, is regarded as "exact"; DLNS results for $8 \leq F \leq 13$ and $-20 \leq J \leq 0$; and SLNS results for $8 \leq F \leq 13$, shown in the last column. For $J$ near 0 , the RMS appears to depend only on the choice of $J$. When $J<-E_{0}$, the RMS for DLNS appears asymptotic to the RMS for the $F$-bit underflow-free SLNS.

For comparision, instead of a simple underflow-free SLNS, we modeled an SLNS which abruptly underflows at $b^{-J}$. Figure 3 shows the RMS error for the same FFT simulation 


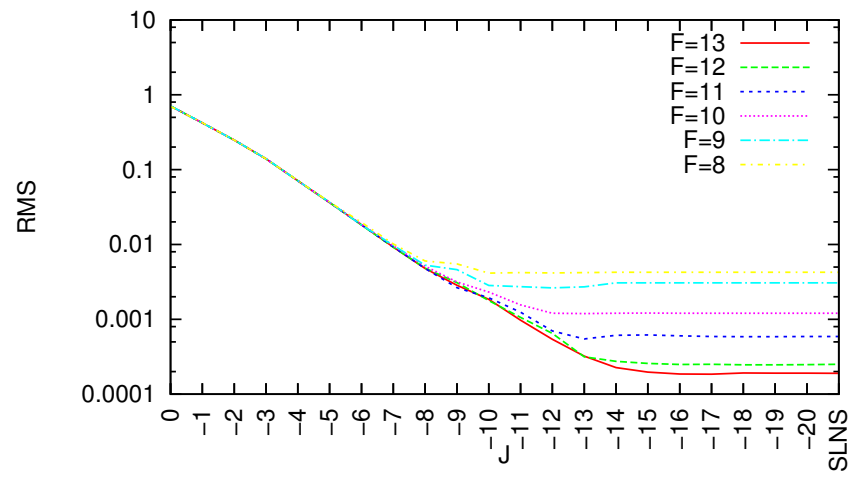

Fig. 2 64-point FFT RMS using actual DLNS and SLNS arithmetic.

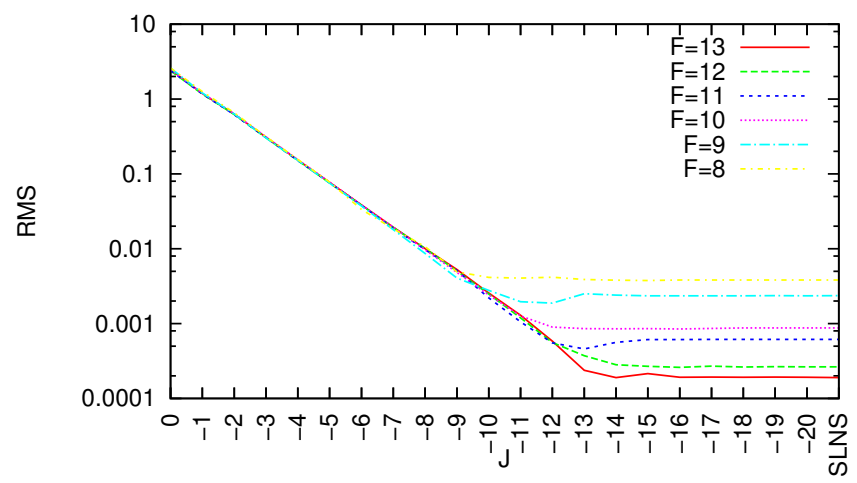

Fig. 3 64-point FFT RMS using abrupt-underflow SLNS arithmetic.

using this abrupt-underflow SLNS. The shape of the curves in Figures 2 and 3 are similar, reaching similar asymptotes; however, for $J$ near zero, DLNS is two to three times more accurate.

We also modeled the DLNS error mechanism more abstractly by injecting noise into each double-precision-FFT step from a random distribution whose width is given by (26). Although Figure 4 is noisy and overestimates the error, it appears similar to the actual simulation results in Figure 2, suggesting (26) is a reasonable model for DLNS behavior.

In some applications, Paliouras and Stouraitis [25] have shown SLNS reduces dynamic power consumption of memory accesses because of decreased switching activity on the memory bus resulting from the compression inherent in the logarithmic representation. To see whether DLNS has similar advantages, we measured switching activity during the memory access pattern of our FFT simulation using actual DLNS arithmetic, and also, for comparision, using SLNS arithmetic. The data are plotted in Figure 5 as a percentage of SLNS switching activity. As is most natural, Figure 5 uses two's complement integers to represent the DLNS $X_{D}$ and SLNS $X_{L}$. This means negative $X_{D}$ represents absolute values less than $1-b^{J}$; for $J=0, X_{D} \geq 0$, which is significant since one of the major causes of increased switching activity is alternating between positive and negative two's complement values in memory. Values of $J$ near zero offer up to $15 \%$ reduction in switching activity; 


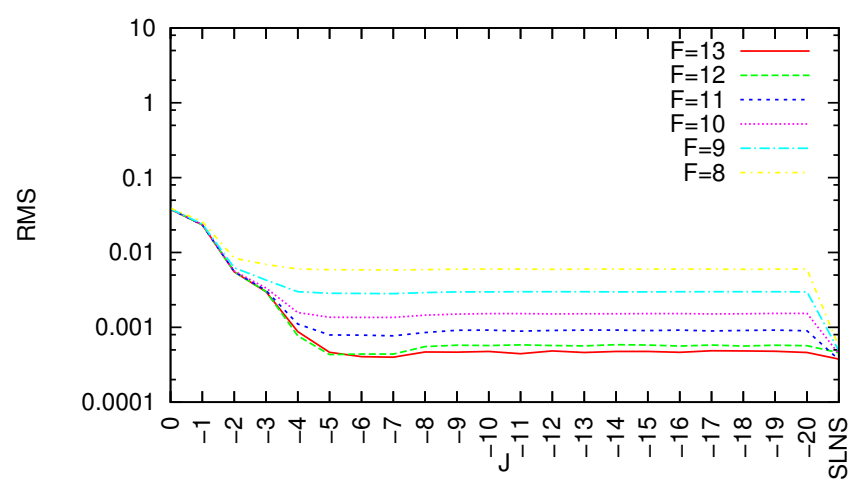

Fig. 4 64-point FFT RMS using random error model (26).

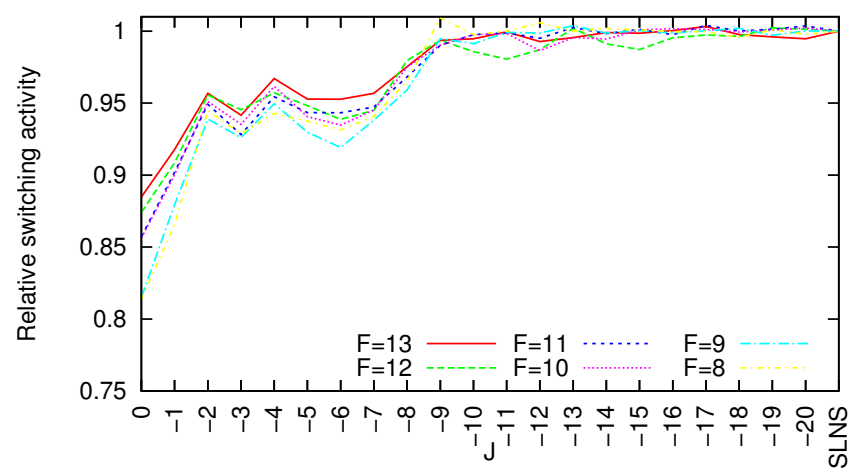

Fig. 5 DLNS FFT Switching Activity (\% SLNS) using Two's Complement $X_{D}$.

$J=-F$ yields a $3 \%$ reduction in switching activity. As $J$ moves further away from zero, the switching activity becomes similar to SLNS.

An alternative to two's complement negative $X_{D}$ is to use an offset (by $J$ ) representation for $X_{D}$, analogous to how IEEE-754 exponents are encoded. Figure 6 shows this offers switching reduction over a wide range of $J$. It reaches $15 \%$ reduction for $J=-8, F=8$ and nearly $25 \%$ reduction for $J=1, F=8$. It is also possible to use offset representation for abrupt-underflow SLNS. Figure 7 shows this offers less switching reduction (around $10 \%$ ) than DLNS, and, as described earlier, this comes at a cost of greater RMS error than DLNS.

To measure software-implementation cost, a 32-bit $(F=23) \mathrm{C}++$ implementation of abrupt-underflow LNS (using interpolation and cotransformation with range and precision comparable to IEEE-754 single precision) was extended to DLNS using $R$ and $T$. A simple computation (described in Section 6.4) was benchmarked on a 1.3GHz Core 2 Duo, using $\mathrm{g}++$ and Microsoft compilers. DLNS only adds around $16 \%$ overhead with purely normal data, because these pass through the extra $s_{b}$ or $d_{b}$ as essential identities. As $k$ moves into the denormal range, the speed of DLNS can be as much as 2.5 times slower than LNS. 


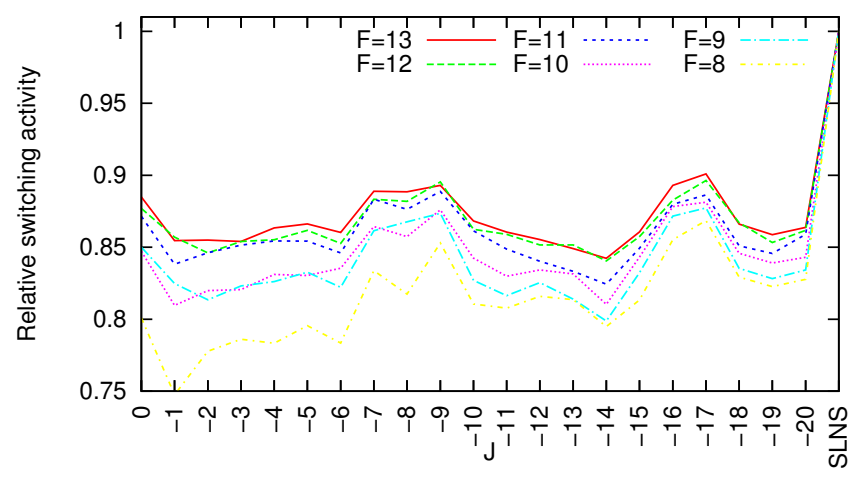

Fig. 6 DLNS FFT Switching Activity (\% SLNS) using Offset $X_{D}$.

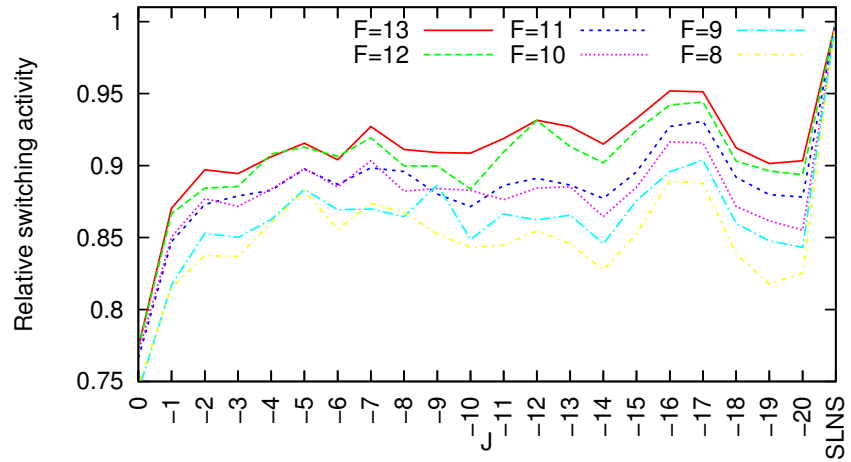

Fig. 7 Abrupt-Underflow SLNS FFT Switching Activity (\% SLNS) using Offset $X_{L}$.

\section{DLNS Synthesis}

We implemented two versions of the proposed DLNS/SLNS ALU designs inside the FloPoCo arithmetic core generator framework. FloPoCo [10] is a software tool that automatically generates arithmetic cores in synthesizable VHDL. It includes support for SLNS arithmetic. Our first ALU implementation computes $T, R^{\prime}, R_{D}^{\prime \prime}, T_{D}^{\prime \prime}$ and $P_{D}$, and our second implementation additionally supports multiply-accumulate operations $P_{D}^{\prime}$ and $Q_{D}$. We leverage the implementations of $s_{b}$ and $d_{b}$ that FloPoCo provides for SLNS. The implementation of $s_{b}$ is based on an optimized polynomial evaluator [11] and $d_{b}$ is evaluated using co-transformation [4].

We synthesized, placed and routed both units for a Xilinx Virtex-4 LX-25 FPGA using the Xilinx ISE 12.3 synthesis toolchain. Table 2 shows the area in FPGA slices and DSP blocks and the combinatorial latency in nanoseconds after place and route, for various precisions. These results are compared with the resources taken by $s_{b}$ and $d_{b}$ alone, a valid point of comparison for typical applications where the signs of numbers are not known. As can be seen, $s_{b}$ and $d_{b}$ account for most of the area and delay. The overheads added by the combined ALU and multiply-accumulate ALU over a conventional SLNS ALU are respectively $26 \%$ and $43 \%$ in the worst case (for $F=10$ ).

Although we synthesized combinatorial versions of the operators for direct delay comparison, all circuits can be pipelined. We expect the additional logic for DLNS would in- 
Table 2 Area and combinatorial delay of synthesized DLNS operators, after place and route. Area is given in general-purpose slices and DSP48 slices. The area and delay of the $s_{b}$ and $d_{b}$ parts synthesized separately are shown for reference. For all entries, $J=3$.

\begin{tabular}{||c|c|c|c|c||c|c|c|c||}
\hline \hline $\mathrm{F}$ & \multicolumn{2}{|c|}{ Combined } & \multicolumn{2}{c||}{$s_{b}$ and $d_{b}$} & \multicolumn{2}{c|}{ Multiply-accumulate } & \multicolumn{2}{c||}{$d_{b}$ and $s_{b}-d_{b}$} \\
& Area & ns & Area & ns & Area & ns & Area & ns \\
\hline 8 & $895+0$ & 35.7 & $739+0$ & 30.9 & $1184+0$ & 42.90 & $892+0$ & 38.6 \\
9 & $1105+0$ & 39.3 & $857+0$ & 31 & $1414+0$ & 46.70 & $1086+0$ & 40.4 \\
10 & $1177+3$ & 42.3 & $936+2$ & 32.8 & $1694+3$ & 50.72 & $1182+2$ & 40 \\
11 & $1462+3$ & 43.0 & $1226+2$ & 34.9 & $1975+3$ & 52.72 & $1686+2$ & 42.2 \\
12 & $1665+6$ & 46.4 & $1479+6$ & 37.8 & $2602+6$ & 54.18 & $1824+6$ & 46.2 \\
13 & $2063+6$ & 46.8 & $1740+6$ & 39.5 & $3279+6$ & 56.53 & $2366+6$ & 53.4 \\
14 & $2356+6$ & 44.6 & $2158+6$ & 37.8 & $4209+6$ & 57.69 & $3004+6$ & 47.4 \\
\hline \hline
\end{tabular}

Table 3 Area and combinatorial delay of synthesized DMLNS and DOMLNS 5-operation ALUs, compared to SLNS. Settings are the same as in table 2.

\begin{tabular}{||c|c|c|c|c|c|c||}
\hline \hline F & \multicolumn{2}{|c|}{ SLNS $\left(s_{b}-d_{b}\right)$} & \multicolumn{2}{c|}{ DMLNS } & \multicolumn{2}{c||}{ DOMLNS } \\
& Area & ns & Area & ns & Area & ns \\
\hline 8 & $509+0$ & 21.852 & $685+1$ & 40.660 & $593+0$ & 25.221 \\
9 & $588+0$ & 21.773 & $765+1$ & 37.887 & $706+0$ & 28.575 \\
10 & $712+1$ & 24.269 & $907+2$ & 40.859 & $843+1$ & 30.664 \\
11 & $983+1$ & 29.557 & $1193+2$ & 44.213 & $1181+1$ & 34.469 \\
12 & $1112+3$ & 29.244 & $1366+4$ & 47.512 & $1284+3$ & 34.111 \\
13 & $1491+3$ & 30.600 & $1776+4$ & 45.852 & $1688+3$ & 35.806 \\
14 & $1717+3$ & 28.186 & $2064+4$ & 44.223 & $1965+3$ & 37.936 \\
\hline \hline
\end{tabular}

crease pipeline depth, but would not significantly affect the minimum cycle time in a highlypipelined implementation, as it only involves a small table and multiplier and pure combinatorial logic.

\section{Mitchell-Based Alternatives: DMLNS and DOMLNS}

Mitchell [31] proposed two related techniques: one produces an approximation to the logarithm, $\log _{2}(X)$, and the other produces an approximation to the antilogarithm, $2^{x}$. (In this section, we will use $X$ and $x$ as simple algebraic variables, which is different than earlier usage.) Although half a century old, these techniques continue to be frequently used primarily in the way Mitchell intended (as a means of low-cost approximate multiplication) [32-38], although a few modern applications have used it for other approximate computations, such as spam filtering [39,40] or ultrasound imaging [41]. Mitchell's methods are approximately equal to ordinary real-valued functions, but Mitchell's results can be computed as precisely as desired at relatively low cost. The confusion between precision and accurary of Mitchell's method was a point of a little controversy some years ago [42,43]. The novel usage of Mitchell's method in this section obtains accurate results because it is being used only for compression of denormal values, rather than the actual arithmetic on such values. Two alternative approaches are considered, which we will call the Denormal Mitchell LNS (DMLNS) and the Denormal Offset Mitchell LNS (DOMLNS). DMLNS and 
DLNS share that large values have the identical representation as SLNS; DOMLNS sacrifices this compatibility for reduced hardware cost.

\subsection{Review}

We start by considering Mitchell's original technique for a restricted range of arguments. For the argument, $X$, in the range $1 \leq X \leq 2$, Mitchell approximates its logarithm as $\log _{2}(X) \approx M(X)=X-1$. If $X$ is outside this range, it is possible to determine int $\left(\log _{2}(X)\right)$ by finding the position of the leading significant bit in $X$ and then shifting appropriately. For $0<X \leq 1.0, M(X) \leq 0.0$ can be described as follows:

$$
\begin{aligned}
& M(X) \quad=2 X-2, \quad 0.5 \leq X \leq 1.0 \\
& =4 X-3, \quad 0.25 \leq X \leq 0.5 \\
& =8 X-4, \quad 0.125 \leq X \leq 0.25 \\
& =16 X-5, \quad 0.0625 \leq X \leq 0.125 \\
& =2^{n} \cdot X-n-1,2^{-n} \leq X \leq 2^{-n+1} \text {. }
\end{aligned}
$$

The other method proposed by Mitchell is an antilogarithm approximation, $M^{-1}(x)$. For the antilogarithm whose argument, $x$, is in the range $0 \leq x \leq 1$, Mitchell approximates the antilogarithm as $2^{x} \approx M^{-1}(x)=x+1$. A simple shift deals with arguments outside this range so that in general

$$
2^{x} \approx M^{-1}(x)=2^{\operatorname{int}(x)} \cdot(\operatorname{frac}(x)+1),
$$

where $\operatorname{int}(X)$ is the unique integer such that $\operatorname{int}(X)+\operatorname{frac}(X)=X$ and $0 \leq \operatorname{frac}(X)<1$. Although not specified by Mitchell, this generalizes to negative $-n<x \leq 0$ :

$$
\begin{array}{rlrl}
M^{-1}(x) & =(x+1) \cdot 0.5+0.5, & -1 \leq x \leq 0 \\
& =(x+2) \cdot 0.25+0.25, & & -2 \leq x \leq-1 \\
& \cdots & & \\
& =(x+n) \cdot 2^{-n}+2^{-n}, & & -n \leq x \leq-n+1 .
\end{array}
$$

The Mitchell logarithm and antilogarithm are exact inverses of each other, $M\left(M^{-1}(x)\right)=x$ and $M^{-1}(M(X))=X$, a property important to their usage in this section.

In addition to its usage as an approximation for the base-two antilogarithm, Mitchell's method has been suggested to approximate the base-two addition logarithm [44-46] as $s_{2}(z) \approx M^{-1}(z)$ for $z<0$. This approximation is exact at $z=0$ and as $z$ approaches $-\infty$ (the expected essential zero property). It is slightly larger otherwise, allowing for approximately 4-bit accuracy. From (7), the case for $z \geq 0$ is $s_{2}(z) \approx z+M^{-1}(-z)$. Although it was also suggested [44] to approximate the subtraction logarithm in a similar way, the two functions would not be exact inverses of each other, and therefore not suitable for use here. 


\subsection{DMLNS}

In DMLNS (unlike [44]), Mitchell's method is not used for the actual computation of sums and differences, which are computed as in ordinary SLNS using interpolated $s_{b}$ and $d_{b}$ units. Instead, Mitchell's method is used in place of (13) in the definition of the denormal decompression function:

$$
\begin{aligned}
f(x) & =J+M^{-1}(x-J) & & x<J \\
& =J+x+M^{-1}(-x-J) & & x \geq J .
\end{aligned}
$$

To simplify the algebra, let's consider the typical case of $J=0$ (analogous results hold for arbitrary $J)$, and introduce for this subsection $n=-\operatorname{int}(-x)=\operatorname{int}(x)+1$ and $y=f(x)$ as the ordinate to the corresponding abcissa, $x$. Inverting (31) in the case $J=0$ and $y<1$ is trivially $f^{-1}(y)=M(y)<0$, as described in the last section.

The novel aspect of inverting (31), which has not been considered in the literature, is when $y \geq 1$. In this case, we are trying to invert $y=x+M^{-1}(-x)$, which from (29) is

$$
y=x+(-x+n) \cdot 2^{-n}+2^{-n} .
$$

In this case, $2^{-n}<1$, causing a right shift by $n$ places. The larger $x$ is, the closer $y$ becomes to $x$, just like $s_{b}(x)$ for $x \gg 0$. The signs of $-x$ and $n$ are opposite, having the effect of computing frac $(-x)$ with the $(-x+n)$.

Solving equation (32) for $x$ :

$$
y=\frac{2^{n}-1}{2^{n}} \cdot x+\frac{n+1}{2^{n}}
$$

we obtain

$$
x=f^{-1}(y)=\frac{2^{n}}{2^{n}-1} \cdot y-\left(\frac{2^{n}-2-n}{2^{n}-1}+1\right) .
$$

The problem is that the $n$ in (33) is defined using the desired result, $x$. We can overcome this by noting that the $y$ endpoints of each interval (in which $n$ remains the same) are of the form $n+2^{-n}$. If $y \geq \operatorname{int}(y)+2^{-\operatorname{int}(y)}$, we use $n=\operatorname{int}(y)+1$; otherwise, we use $n=\operatorname{int}(y)$.

\subsection{DOMLNS}

DMLNS requires computing the reciprocal of odd constants, and this can be somewhat expensive in hardware[48,49]. If we are willing to sacrifice bit-level compatibility to SLNS (and also accept a reduction in the largest representable value by half), we could offset the decompression function so that the multiplication by $2^{n} /\left(2^{n}-1\right)$ is unnecessary. Instead, with Denormal Offset Mitchell LNS (DOMLNS), a non-denormal value is represented by a fixed-point representation which is one larger than the fixed-point representation that would be used in SLNS:

$$
\begin{aligned}
f(x) & =J+M^{-1}(x-J) & & x<J \\
& =x+1 & & x \geq J .
\end{aligned}
$$

The inverse of this is easily computed

$$
\begin{aligned}
f^{-1}(x) & =J+M(x-J) & & x<J+1 \\
& =x-1 & & x \geq J+1 .
\end{aligned}
$$


6.4 Taylor-series Benchmarks

In order to experiment with the variations of logarithmic denormal representation proposed here, several truncated-Taylor-series values were computed using 32-bit words $(F=23$ bit precision) after scaling the terms by $2^{-i}$, where the range $100 \leq i \leq 145$ was chosen to start well before subnormals would be encountered in the summation and end with most subnormal terms underflowing to zero. These results may be compared against IEEE-754 single precision FP. Figure 8 shows the relative errors of FP, SLNS, DLNS, DMLNS and DOMLNS when computing the non-negative series

$$
2^{-i} \cdot e^{1} \approx \sum_{k=0}^{10} \frac{2^{-i}}{k !}
$$

Figure 9 shows similar results for the alternating series

$$
2^{-i} \cdot \sin (\pi / 4)=2^{-i} \sqrt{0.5} \approx \sum_{k=0}^{10}(-1)^{k} \frac{2^{-i}(\pi / 4)^{2 k-1}}{(2 k-1) !}
$$

Figure 10 works with a larger number of terms of the slower converging alternating series

$$
2^{-i} \cdot \arctan (1)=2^{-i} \cdot \pi / 4 \approx \sum_{k=0}^{100}(-1)^{k} \frac{2^{-i}}{(2 k-1)} .
$$

The alternating series force the arithmetic to deal with sums and differences; the $e$-series avoids some of those complications. In each figure, SLNS fails to produce any usable result once the first term of the series falls below the denormal boundary, and SLNS results are not shown in these cases. Even when the correct answer has a valid non-denormal representation, the plots show the relative errors grow much larger than for any of the systems which can represent denormal values. This is because denormals capture some of the information from the higher-order terms of the Taylor series, which the truncation behavior of SLNS is unable to do.

The behavior of all techniques proposed roughly matches that of IEEE-754 for subnormal values, although in this range DLNS is often as or more accurate than FP. DOMLNS and DMLNS, on the other hand, are often identical to each other but slightly less accurate than FP.

DMLNS has problems with the quickly converging alternating sine series prior to the denormal boundary, where it should be as accurate as FP and SLNS. We conjecture that since it is impossible to approximate $2^{n} /\left(2^{n}-1\right)$ perfectly for DMLNS, non-denormal values are biased during repeated applications of $f$ and $f^{-1}$.

\subsection{DMLNS and DOMLNS Synthesis}

To evaluate the overhead of DMLNS and DOMLNS, we consider a combined arithmetic unit performing either addition, subtraction, multiplication, division and square root. In the DMLNS and DOMLNS cases, two compression functions and one decompression function are shared between all operators. Table 3 compares the resource usage of synthesized 5-operation arithmetic units in SLNS, DMLNS and DOMLNS. Adding compression and decompression functions to the inputs and output of SLNS operators leads to an area overhead 


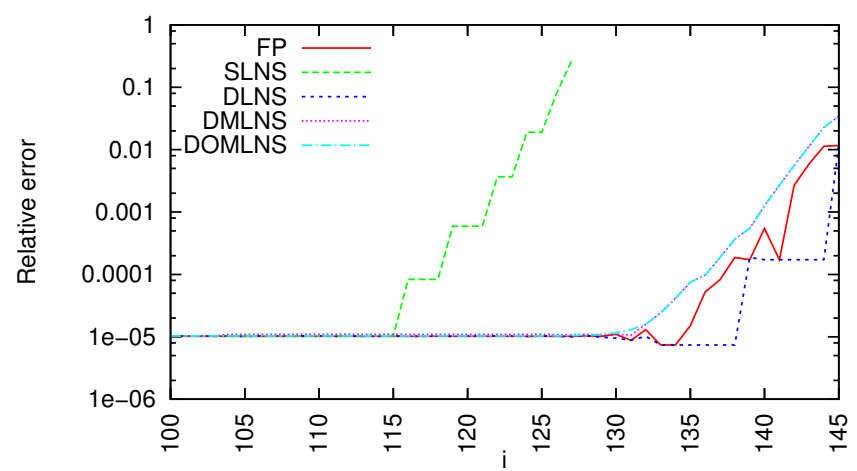

Fig. 8 Relative error in ten-term $2^{i}$-scaled Taylor series for $e$.

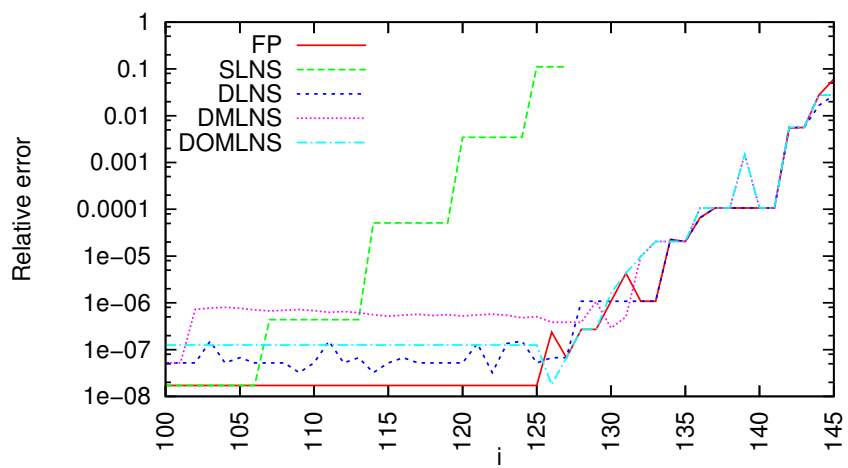

Fig. 9 Relative error in ten-term $2^{i}$-scaled Taylor series for $\sin (\pi / 4)$.

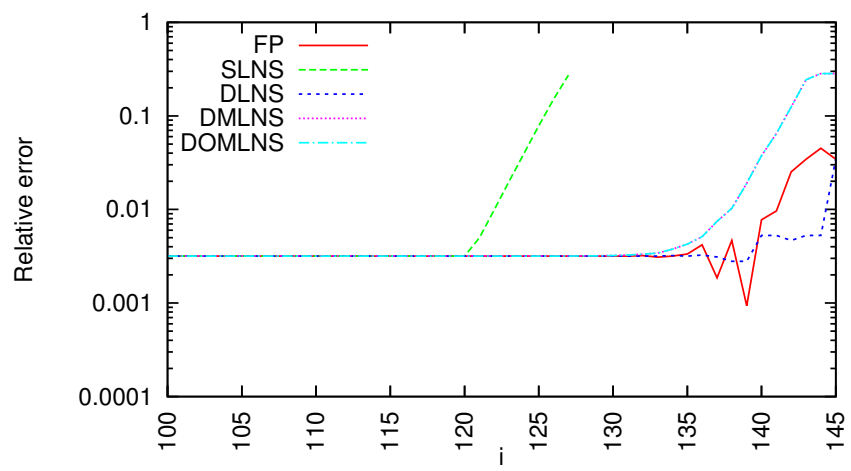

Fig. 10 Relative error in 100-term $2^{i}$-scaled Taylor series for $\arctan (1)$. 
comprised between $19 \%(\mathrm{~F}=13)$ and $35 \%(\mathrm{~F}=8)$ using DMLNS, and between $13 \%(\mathrm{~F}=13)$ and $20 \%(\mathrm{~F}=9)$ using DOMLNS. The implementation of each DMLNS $f$ function also consumes a DSP slice, while DOMLNS is implemented in logic only. DMLNS has a substantial cost in latency (between 50\% and 86\%), while DOMLNS has a much lower impact (between $15 \%$ and $35 \%$ ). As the implementation of $f$ and $f^{-1}$ scale better with precision than SLNS addition, higher precisions tend to amortize the overhead of DMLNS and DOMLNS.

\section{Conclusions}

This paper introduced three new alternatives for including gradual underflow in logarithmic arithmetic: the Denormal Logarithmic Number System (DLNS), the Denormal Mitchell LNS (DMLNS) and the Denormal Offset Mitchell LNS (DOMLNS). Large values have identical representations in DLNS and DMLNS as they have in the conventional Signed LNS (SLNS). DLNS, DMLNS and DOMLNS are hybrids of the properties of the FiXedpoint Number System (FXNS) and SLNS, that can be characterized in terms of base (typically $b=2$ ), precision $(F)$ and a new design parameter, $J$, which allows customizing the range in which gradual underflow occurs. $J=0$ gives a wide gradual underflow range that acts like FXNS (and like the $\mu$ law); $J<-F$ gives a narrow gradual underflow range that act likes SLNS (and like the IEEE-754 standard). Taylor-series computation of common mathematical constants scaled to be in the denormal range show that when $F$ and $J$ are chosen as in the IEEE-754 Floating-Point (FP) standard, DLNS, DMLNS and DOMLNS have similar gradual-underflow behavior as FP.

DLNS is most suitable for application-specific problems that only require add, subtract and mixed multiply (where one operand can be preconverted to SLNS). An example of such an application is the Fast Fourier Transform (FFT). Simulation of an FFT application illustrates DLNS using $J \approx 0$ decreases bit-switching activity $15 \%$ with a two's complement encoding and nearly $25 \%$ with an offset representation; however, this causes significant increase in RMS error. A choice of $J=-F$ provides a balanced design point, decreasing bit-switching activity by $15 \%$ with an offset representation at the cost of a $30 \%$ increase in RMS error. DLNS reduces switching activity 5\% to $20 \%$ more than an abruptlyunderflowing SLNS with around one-half the RMS error. The majority of the area of the synthesized DLNS circuit is for traditional SLNS addition and subtraction tables; only a small area is used for the novel datapaths that allow the ALU to act on conventional SLNS as well as DLNS and mixed data. Although DLNS is affordable for such application-specific situations, the question of its suitability for general-purpose computation is problematic. In software, general-purpose DLNS only increases overhead about $16 \%$ for computations like the Taylor-series examples involving non-denormal data, with signifcant overhead only for denormal data. In hardware, general-purpose DLNS requires multiple instances of the traditional SLNS addition and subtraction tables which make the hardware very expensive. While general multipliction and division in SLNS are inexpensive, they are are very expensive in DLNS.

For applications that need general multiplication and division, this paper proposed two other novel variations (DMLNS and DOMLNS) that simplify hardware using Mitchell's method so that the cost of general multiplication and division is much lower. DMLNS and DOMLNS offer tradeoffs that allow for further reduction in area, at the expense of a minor decrease in accuracy for numbers in the denormal range. DOMLNS sacrifices compatibility with SLNS representations of large values in order to lower hardware cost and to improve accuracy compared to DMLNS. 


\section{Acknowledgements}

We wish to thank the reviewers from their insightful comments and suggestions.

\section{References}

1. M. G. Arnold, T. A. Bailey, J. R. Cowles and J. J. Cupal, "Redundant Logarithmic Arithmetic," IEEE Trans. Comput., vol. 39, pp. 1077-1086, Aug. 1990.

2. M. G. Arnold, T. A. Bailey, J. R. Cowles and M. D. Winkel, "Applying Features of IEEE 754 to Sign/Logarithm Arithmetic," IEEE Trans. Comput., vol. 41, pp. 1040-1050, Aug. 1992.

3. M. Arnold and C. Walter, "Unrestricted Faithful Rounding is Good Enough for Some LNS Applications," 15th Intl. Symp. Computer Arithmetic, Vail, Colorado, pp. 237-245, 11-13 June 2001.

4. M. Arnold and C. Collange, "A Dual-Purpose Real/Complex Logarithmic Number System ALU," 19th Intl. Symp. Computer Arithmetic, Portland, OR, pp. 15-24, 8-10 June 2009.

5. M. Arnold, et al. "Towards a Quaternion Complex Logarithm Number System," 20th Intl. Symp. Computer Arithmetic, Tuebingen, Germany, pp. 33-42, 25-27 July 2011.

6. C. Chen and C. H. Yang, "Pipelined Computation of Very Large Word-Length LNS Addition/Subtraction with Polynomial Hardware Cost," IEEE Trans. Comput., vol. 49, no. 7, pp. 716-726, July 2000.

7. E. I. Chester and J. N. Coleman, "Matrix Engine for Signal Processing Applications Using the Logarithmic Number System," Proceedings of the IEEE Intl. Conf. on Application-Specific Systems, Architectures and Processors, San Jose, California, pp. 315-324, 17-19 July 2002.

8. J. N. Coleman, E. I. Chester, C. I. Softley, and J. Kadlac, "Arithmetic on the European Logarithmic Microprocessor," IEEE Trans. Comput., vol. 49, no. 7, pp. 702-715, July 2000.

9. J. N. Coleman, C. I. Softley, J. Kadlec, R. Matousek, M. Tichy, Z. Pohl, A. Hermanek, and N. F. Benschop, "The European Logarithmic Microprocessor", IEEE Transactions on Computers, pp. 532-546, 2008.

10. F. de Dinechin, "The Arithmetic Operators You Will Never See in a Microprocessor", 20th Intl. Symp. Computer Arithmetic, Tuebingen, Germany, pp, 189-190, July 2011.

11. F. de Dinechin, M. Joldes and B. Pasca, "Automatic Generation of Polynomial-Based Hardware Architectures for Function Evaluation", Application-specific Systems, Architectures and Processors, IEEE, 2010.

12. A.D. Edgar and S.C. Lee, "FOCUS Microcomputer Number System," Commun. of the ACM, vol. 22, p. 166-167, 1979.

13. H. Fu, O. Mencer and W. Luk, "FPGA Designs with Optimized Logarithmic Arithmetic", IEEE Transactions on Computers, vol. 59, no. 7, pp. 1000-1006, July 2010.

14. K. F. Gauss, Werke, vol. 8, pp. 121-128, 1900.

15. IEEE Standard for Binary Floating-Point Arithmetic, ANSI/IEEE Std 754-1985, IEEE, 1985.

16. IEEE Standard for Floating-Point Arithmetic, ANSI/IEEE Std 754-2008, IEEE, 2008.

17. R. C. Ismail and J. N. Coleman, "ROM-less LNS", 20th Intl. Symp. Computer Arithmetic, Tuebingen, Germany, pp. 43-51, July 2011.

18. S. J. Kidd, "Implementation of the Sign-Logarithm Arithmetic FFT,"Royal Signals and Radar Establishment Memorandum 3644, Malvern, 1983.

19. N. G. Kingsbury and P. J. W. Rayner, "Digital Filtering Using Logarithmic Arithmetic," Electron. Lett., vol. 7, no. 2, pp. 56-58, Jan 28, 1971.

20. M. Kahrs and K. Branderburg, Editors., Applications of Digital Signal Processing to Audio and Acoustics, Kluwer Academic Publ., Norwell, Massachusetts, p. 224, 1998.

21. D. M. Lewis, "114 MFLOPS Logarithmic Number System Arithmetic Unit for DSP Applications," Intl. Solid-State Circuits Conf., San Francisco, pp. 1547-1553, Feb. 1995.

22. V. S. Dimitrov, J. Eskritt, L. Imbert, G. A. Jullien and W. C. Miller, "The Use of The Multi-Dimensional Logarithmic Number System in DSP Applications," 15th Intl. Symp. Computer Arithmetic, Vail, Colorado, pp. 247-254, 11-13 June 2001.

23. I. Kouretas, Ch. Basetas and V. Paliouras, "Low-Power Logarithmic Number System Addition and Subtraction and their Impact on Digital Filters," IEEE Trans. Comput., 29 May 2011, IEEE Computer Society Digital Library, http://doi.ieeecomputersocety.org/10.1109/TC.2012.111

24. Junichiro Makino and Makoto Taiji, Scientific Simulations with Special-Purpose Computers-the GRAPE Systems, John Wiley and Sons, Chichester, England, 1998.

25. V. Paliouras and T. Stouraitis, "Low Power Properties of the Logarithmic Number System," Proceedings of the 15th IEEE Symp. on Computer Arithmetic, Vail, Colorado, pp. 229-236, 11-13 June 2001. 
26. E. E. Swartzlander, D. Chandra, T. Nagle, and S. A. Starks, "Sign/logarithm Arithmetic for FFT Implementation," IEEE Trans. Comput., vol. C-32, pp. 526-534, 1983.

27. E. E. Swartzlander and A. G. Alexopoulos, "The Sign/Logarithm Number System," IEEE Trans. Comput., vol. C-24, pp. 1238-1242, December 1975.

28. S. Young, et al., The HTK Book (for HTK Version 3.1), Cambridge University Engineering Department, England, Dec. 2001 http://htk.eng.cam.ac.uk

29. www.xlnsresearch. com has an extensive bibliography of LNS-related articles.

30. "Pulse Code Modulation (PCM) of Voice Frequencies", International Telecomunications Union, 1988. http://www.itu.int/rec/T-REC-G.711/en

31. J. N. Mitchell, "Computer Multiplication and Division using Binary Logarithms," IEEE Trans. Electronic Comput., vol. EC-11, pp. 512-517, August 1962.

32. Khalid H. Abed and R. E. Siferd, "CMOS VLSI Implementation of a Low-Power Logarithmic Converter," IEEE Transactions on Computers, vol. 52, no. 11, pp. 1421-1433, Nov. 2003.

33. Khalid H. Abed and R. E. Siferd, "VLSI Implementation of a Low-Power Antilogarithmic Converter," IEEE Transactions on Computers, vol. 52, no. 9, pp. 1221-1228, Sept. 2003.

34. Satish Bhairannawar et al., "FPGA based Recursive Error-Free Mitchell Log Multiplier for Image Filters," IEEE International Conference on Computational Intelligence and Computing Research (ICCIC), Coimbatore, India, pp. 1-5, 18-20 Dec. 2012. doi: 10.1109/ICCIC.2012.6510248

35. V. Mahalingam and N. Ranganathan, "Improving Accuracy in Mitchell's Logarithmic Multiplication Using Operand Decomposition," IEEE Transactions on Computers, vol. 55, no. 12, pp. 1523-1535, Dec. 2006.

36. D.J. McLaren, "Improved Mitchell-Based Logarithmic Multiplier for Low-power DSP Applications," IEEE International System On Chip (SOC) Conference, pp. 53-56, 17-20 Sept. 2003.

37. M. Sullivan and E. E. Swartzlander "Truncated Logarithmic Approximation", 21th Symposium on Computer Arithmetic, Austin, TX, Apr. 2013.

38. D.R. Shetty and S. Patil, "Improving Accuracy in Mitchell's Logarithmic Multiplication Using Iterative Multiplier for Image Processing Application", International Journal of Soft Computing and Engineering (IJSCE) ISSN: 2231-2307, Vol. 3, No. 3, pp. 187-191, July 2013.

39. C. Layer, H. J. Pfleiderer and C. Heer, "A Scalable Compact Architecture for the Computation of Integer Binary Logarithms through Linear Approximation," 2004 International Symposium on Circuits and Systems (ISCAS), vol. 2, pp. 421-424, Vancouver, Canada, 23-26 May 2004.

40. M. N. Marsono, M. W. El-Kharashi and F. Gebali, "Binary LNS-based Naïve Bayes Hardware Classifier for Spam Control," IEEE International Symposium on Circuits and Systems (ISCAS), Kos, Greece, pp. 3674 - 3677, 21-24 May 2006.

41. A. Page and T. Mosemnin, "An Efficient and Reconfigurable FPGA and ASIC Implementation of a Spectral Doppler Ultrasound Imaging System”, 21th Symposium on Computer Arithmetic, Austin, TX, Apr. 2013.

42. R. Maenner, “A Fast Integer Binary Logarithm of Large Arguments,” IEEE Micro, vol. 7, no. 6, pp. 41-45, Dec. 1987

43. M. Arnold, T. Bailey, J. Cowles and J. Cupal, "Error Analysis of the Kmetz/Maenner Algorithm," Journal of VLSI Signal Processing, vol. 33, pp. 37-53, Oct. 2002.

44. M. G. Arnold, "LPVIP: A Low-power ROM-Less ALU for Low-Precision LNS," 14th International Workshop on Power and Timing Modeling, Optimization and Simulation, LNCS 3254, pp. 675-684, Santorini, Greece, 15-17 Sept. 2004.

45. M. G. Arnold and P. Vouzis, "A Serial Logarithmic Number System ALU," EuroMicro Digital System Design DSD, Lubeck, Germany, pp. 151-156, 29 Aug. 2007.

46. M.G. Arnold, "Improved DNA-sticker Arithmetic: Tube-encoded-carry, Logarithmic Number System and Monte-Carlo methods," Natural Computing, Vol. 12, no. 2, pp. 235-246, 2013.

47. M. G. Arnold, and C. Collange, "The Denormal Logarithmic Number System",24th International Conference on Application Specific Systems, Architectures and Processors (ASAP), Washington, DC, June 2013

48. S.-Y. R. Li, "Fast Constant Division routines," IEEE Trans. Comput., vol. C-34, pp. 866-869, Sept. 1985

49. D. H. Jacobsohn, "A Combinatoric Division Algorithm for Fixed-Integer Division", IEEE Trans. Comput., vol. C-22, pp. 608-610, June 1973. 